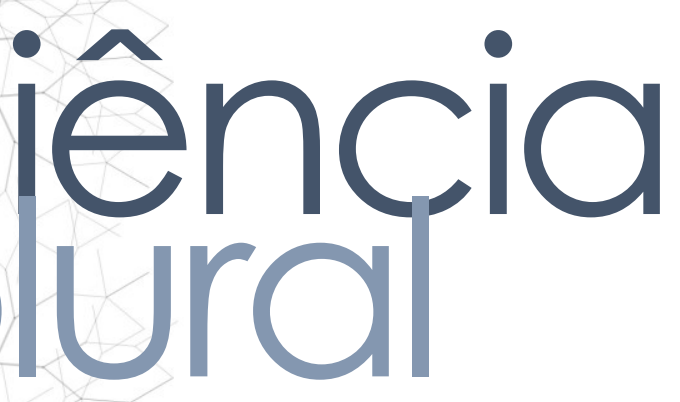

\title{
COMO ESTRUTURAR UM CURRÍCULO INTEGRADO NUM CURSO DE ODONTOLOGIA?
}

How to structure an integrated curriculum in a Dentistry Course?

Luiz Noro || Universidade Federal do Rio Grande do Norte || Professor do Departamento de Odontologia e do Programa de Pós-graduação em Saúde Coletiva | | E-mail: noro@ufrnet.br

Autor responsável pela correspondência

Luiz Noro | | E-mail: noro@ufrnet.br 


\section{RESUMO}

Introdução: Apesar do advento das Diretrizes Curriculares Nacionais (DCN) dos cursos de Odontologia desde 2002, o desenho dos currículos de grande parte dos cursos ainda mantém disciplinas isoladas, sem articulação com os elementos essenciais para a formação de generalistas, com distanciamento entre o ciclo básico e o profissionalizante. Objetivo: Desenvolver proposta de currículo integrado, mediado por metodologias ativas de aprendizagem e avaliação processual da aprendizagem. Método: Para construção da proposta foram identificados eixos sobre os quais foi formulado currículo integrado coerente com as DCN. Resultados: O currículo é composto por cinco eixos: Módulos integrados, Estágio supervisionado, Extensão curricular, Flexibilização e Trabalho de Conclusão do Curso. Os Módulos integrados articulam habilidades e competências que envolvem conhecimentos das áreas básica, clínica e saúde coletiva. O Estágio supervisionado ocorre ao longo dos dois últimos períodos do curso, no Sistema Único de Saúde. A Extensão curricular configura-se pelo desenvolvimento de atividades que articulam a inserção da comunidade com o aprendizado dos alunos, buscando benefícios para o entorno do curso. Para a Flexibilização foram definidos: (1) Atividades complementares e (2) Componentes curriculares optativos, permitindo ao aluno definição do percurso formativo singular. O Trabalho de Conclusão do Curso deve ser desenvolvido de forma transversal desde o primeiro semestre do curso, por componentes curriculares específicos relacionados ao aprendizado das metodologias da pesquisa científica ou nos próprios módulos integrados. Conclusões: Para viabilizar o desenvolvimento do currículo é fundamental a adoção de metodologias ativas de aprendizagem em todos os eixos previstos, permitindo que o aluno construa seu aprendizado. Da mesma forma é fundamental processo de avaliação que contribua efetivamente com uma relação professor-aluno mediada pela busca de conhecimento, permitindo que ambos sejam sujeitos no processo. $\mathrm{O}$ principal desafio para alcance da proposta refere-se ao processo permanente de desenvolvimento docente na construção de uma "nova" sala de aula.

Palavras-Chave: Currículo / Aprendizagem Baseada em Problemas / Avaliação Educacional / Desenvolvimento de pessoal 


\begin{abstract}
Introduction: Despite the advent of the National Curricular Guidelines (DCN) of Dentistry courses since 2002, the curriculum design of most courses still maintains isolated disciplines, without articulation with the essential elements for the formation of generalists, with a distance between the cycle basic and professional one. Objective: To develop a proposal for an integrated curriculum, mediated by active learning methodologies and process evaluation of learning. Methods: To construct the proposal, axes were identified to compose an integrated curriculum, coherent with the DCN. Results: The curriculum consists of five axes: Integrated Modules, Supervised Internship, Extension, Flexibilization and Course Completion Work. The Integrated modules articulate skills and competences that involve knowledge of the basic, clinical and collective health areas. The Supervised internship takes place during the last two periods of the course, in the Unified Health System. The Extension is configured by the development of activities that articulate the insertion of the community with the students' learning, seeking benefits for the course environment. For Flexibilization were defined: (1) Complementary activities and (2) Optional curricular components, allowing the student to define the singular formative course. The Course Conclusion Work should be developed transversally from the first semester of the course, by specific curricular components related to the learning of scientific research methodologies or in the integrated modules themselves. Conclusions: In order to make curriculum development, it is fundamental to adopt active learning methodologies in all the planned axes, allowing the student to build his learning. Likewise, it is fundamental to evaluate the evaluation process that effectively contributes to a teacher-student relationship mediated by the search for knowledge, allowing both to be subjects in the process. The main challenge to reach the proposal refers to the permanent process of teacher development in the construction of a "new" classroom.
\end{abstract}

Keywords: Curriculum / Problem-Based Learning / Educational Measurement / Staff Development 


\section{Introdução}

As Diretrizes Curriculares Nacionais (DCN) dos cursos de Odontologia configuraram-se no início do século XXI como um dos maiores avanços na perspectiva da formação de cirurgiões-dentistas efetivamente vinculada à compreensão da realidade social, cultural e econômica brasileiras, apesar das dificuldades ainda sinalizadas ${ }^{1}$. Para isso, propõem uma formação generalista, com abordagem humanista, crítica e reflexiva, apta a desenvolver atividades referentes à saúde bucal da população, a partir de uma perspectiva de transformação da realidade em benefício da sociedade² .

Tais princípios continuam desafiando a maioria dos cursos de Odontologia do Brasil na condução de um processo formativo que permita a real viabilização dessa proposta, sinalizada em grande parte dos projetos pedagógicos, mas com pouca consistência em seu desenvolvimento ${ }^{3}$.

Possivelmente, essa dificuldade tenha relação direta com a dificuldade da construção de um projeto pedagógico construído coletivamente, centrado no aluno como sujeito do aprendizado e no professor como facilitador da aprendizagem, conforme preconizado nas DCN.

Quando assume essa postura, fica clara a opção pedagógica sinalizada nas DCN pelo emprego de metodologias ativas de aprendizagem no processo formativo. Ao optar por esse caminho, torna-se imperiosa a organização de uma estrutura curricular que permita o desenvolvimento dos diversos conhecimentos, competências e habilidades gerais e específicas, por meio de adequada articulação entre teoria e prática, além da integração dos ciclos básico, clínico e de saúde coletiva 4 .

Contrariamente a essas orientações, grande parte dos cursos de Odontologia nantém ainda no desenho de seus currículos disciplinas estanques, as quais são ordadas em momentos específicos do curso. Nessas situações, nos semestres 
iniciais são abordadas as disciplinas tradicionalmente definidas como do "ciclo básico", destacando-se a Anatomia, Histologia, Fisiologia, entre outras, geralmente responsáveis pelo conhecimento do funcionamento normal dos órgãos e sistemas humanos ${ }^{5}$. A compreensão é que somente poder-se-ia compreender os problemas (agravos ou doenças) a partir do conhecimento do "normal". Em alguns currículos, também são incluídas nesse momento do curso disciplinas da área das ciências sociais e humanas (Antropologia, Sociologia, entre outras) na possível perspectiva de aproximação dessa área com uma formação humanista. Esse ciclo se completa na sequência com disciplinas envolvidas com a Patologia (Microbiologia e Imunologia) para compreensão dos agravos e doenças, sendo sequenciado pela Farmacologia, responsável pelos conteúdos que indicam as possíveis intervenções terapêuticas. Passada essa fase, definida pelos alunos no mesmo estudo5, como "rito de passagem", são iniciadas as fases laboratoriais das disciplinas definidas a partir das especialidades odontológicas (Periodontia, Dentística, Endodontia, Cirurgia) e posteriormente a fase clínica, momento no qual os procedimentos "treinados" pelo aluno no laboratório, passam a configurarem-se como resultado de seu aprendizado na boca do paciente.

Considerando a necessidade da integração, observa-se a criação em vários cursos de componentes denominados "módulos integrativos" ou "seminários integrados", com cargas horárias bastante reduzidas, os quais se propõem a possibilitar a integração dos conteúdos das diferentes disciplinas em um mesmo semestre ou em um conjunto de semestres. Sem dúvida, tal estratégia não apresenta consistência no propósito básico de efetivamente se trabalhar com um currículo integrado.

Buscando contribuir com essa construção, o presente estudo tem como objetivo trazer uma proposta de radicalização na integração curricular, sugerindo elementos fundamentais que devem compor um currículo integrado, nsiderando os princípios sinalizados nas abordagens centradas em metodologias 
ativas de aprendizagem e nos requisitos legais previstos no desenvolvimento da educação superior.

\section{Método}

Para construção da proposta foram primeiramente identificadas as exigências sinalizadas nos principais instrumentos legais que definem padrões em relação aos elementos que devem ser contemplados na educação superior, possibilitando a configuração de uma estrutura curricular que contemplasse esses preceitos, tanto na perspectiva de carga horária como das características pedagógicas exigidas.

Também foram consultados artigos científicos e livros que aprofundam a discussão sobre as estratégias que dão suporte a definição de desenho de currículo integrado e sua construção na perspectiva da construção ativa do aprendizado pelo aluno, permitindo a formulação proposta no presente estudo $0^{6,7,8,9}$.

Em relação à carga horária total, trabalhou-se com a perspectiva de um curso com 4000 (quatro mil) horas, carga horária mínima exigida para o desenvolvimento de um curso de Odontologia10. Para a integralização curricular, optou-se por um currículo a ser desenvolvido em 10 semestres, com base no mesmo parecer ${ }^{10}$, permitindo que o processo formativo acompanhe a maturidade necessária para formação de um profissional de saúde. Esses dois elementos são a base para qualquer curso organizar suas atividades didáticas assim como distribuir a carga horária do corpo docente.

Para distribuição da carga horária e dos eixos foi estabelecido um curso com oferta semestral, sendo o período a ser trabalhado definido como semestre letivo. Em seguida, foram observadas a porcentagem de carga horária de determinados ixos, também já definidos em instrumentos legais. Assim, conforme preconizado próprias DCN2, 20\% da carga horária total do currículo foi destinada aos gios curriculares obrigatórios. Em relação à curricularização da Extensão, 


\section{ciência

conforme previsto no Plano Nacional de Educação ${ }^{11}$ é determinado que cada curso deve destinar, pelo menos, $10 \%$ da carga horária para essa estratégia.

Uma vez estabelecidos esses parâmetros, foi definido porcentagem de carga horária para desenvolvimento dos outros eixos sinalizados nas DCN, qual seja, o Trabalho de Conclusão de Curso e as atividades relacionadas com a flexibilidade curricular (atividades complementares e componentes optativos). A partir daí, estabeleceu-se que a carga horária restante para complementação das 4000 (quatro mil) horas seria utilizada para desenvolvimento das atividades relacionadas às competências e habilidades gerais e específicas, representado pelo Módulo integrado.

Considerando tratar-se de uma proposta definida a partir de revisão de literatura e determinados documentos legais, não houve necessidade de submissão do projeto de pesquisa ao Comitê de Ética em Pesquisa.

\section{Resultados}

O desenho do currículo previsto para ser desenvolvido em 10 semestres letivos, com carga horária total de 4000 (quatro mil) horas é composto por cinco grandes eixos: Módulos integrados, Extensão curricular, Flexibilização (Atividades complementares e Componentes optativos), Estágio curricular supervisionado e Trabalho de Conclusão do Curso. A distribuição proporcional de carga horária desses eixos ao longo dos períodos pode ser observada na figura 1. 


\section{ciência \\ plural}

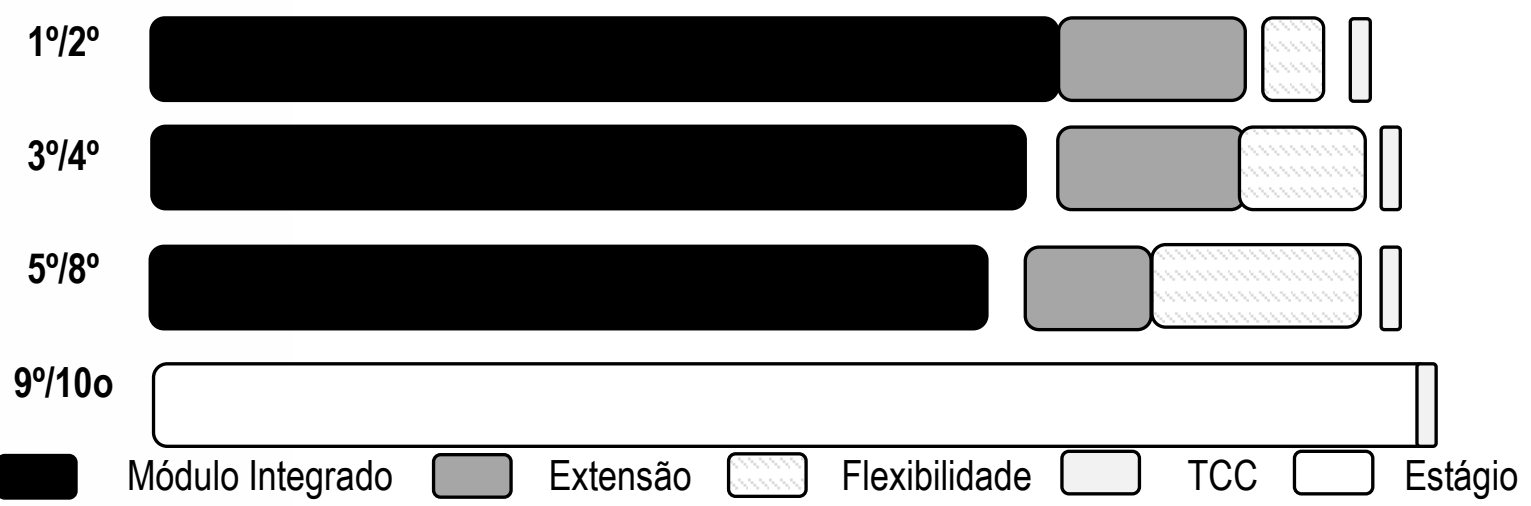

Figura 1. Distribuição proporcional de carga horária dos eixos ao longo dos períodos.

Pode-se observar que do $1^{\mathrm{o}}$ ao $8^{\circ}$ período, o currículo apresenta apenas 4 eixos que devem ser desenvolvidos ao longo do semestre. Com isso, a perspectiva de integração dos conhecimentos apresenta-se privilegiada, uma vez que se evita a fragmentação dos conhecimentos em disciplinas, além de permitirem efetiva articulação com a pesquisa e a extensão. Além disso, existe um equilíbrio bastante adequado em relação a carga horária entre os semestres (máximo 410 horas e mínimo 390), permitindo melhor planejamento tanto para a definição da composição de carga horária por semestre para o corpo docente, como possibilitando ao aluno organização de suas atividades acadêmicas em relação a seus estudos e outras atividades.

Detalhando a proposta considerando cada um dos semestres, é apresentada no Quadro I a carga horária em cada um dos eixos e sua totalização.

Quadiro 1. Total de carga horária em cada eixo por semestre letivo. Natal/RN, 2019

\begin{tabular}{|l|c|c|c|c|c|c|c|c|c|c|c|}
\hline Eixo Período & 1 & 2 & 3 & 4 & 5 & 6 & 7 & 8 & 9 & 10 & T \\
\hline Módulo Integrado & 300 & 300 & 290 & 290 & 280 & 280 & 280 & 280 & & & 2300 \\
\hline Extensão curricular & 60 & 60 & 60 & 60 & 40 & 40 & 40 & 40 & & & 400 \\
\hline $\begin{array}{l}\text { Atividades } \\
\text { Complementares }\end{array}$ & 20 & 20 & 40 & 40 & 20 & 20 & 20 & 20 & & & 200 \\
\hline $\begin{array}{l}\text { Componentes } \\
\text { Optativos }\end{array}$ & & & & & 50 & 50 & 50 & 50 & & & 200 \\
\hline $\begin{array}{l}\text { Estágio curricular } \\
\text { Trabalho de Conclusão } \\
\text { de Curso }\end{array}$ & 10 & 10 & 10 & 10 & 10 & 10 & 10 & 10 & 10 & 10 & 100 \\
\hline TOTAL & 390 & 390 & 400 & 400 & 400 & 400 & 400 & 400 & 410 & 410 & 4000 \\
\hline
\end{tabular}




\section{ciêncíncia
pural}

Nessa distribuição, os Módulos integrados (57,5\% da carga horária total) são desenvolvidos ao longo dos 8 primeiros semestres do curso e integram habilidades e competências que envolvem conhecimentos das áreas básica, clínica e saúde coletiva, caracterizando-se como elementos essenciais para a proposta de se trabalhar com base na Metodologia da Problematização ${ }^{12}$.

Cada semestre apresentará um único Módulo integrado. Esse eixo será o que caracteriza a proposta global de formação do curso, envolvendo todos os professores e sendo o responsável por proporcionar a discussão sobre a forma como as diversas áreas devem se integrar, pensando o desenvolvimento de atividades com aumento de complexidade. Para isso, deve desafiar a criatividade do conjunto de professores e núcleo docente estruturante na efetiva perspectiva de construção de conhecimento, considerando a ainda limitada formação docente didáticopedagógica $^{13}$. Os Módulos integrados devem ser construídos a partir do perfil de egresso previsto no projeto pedagógico, assim como permitir o desenvolvimento das competências e habilidades gerais e específicas.

A forma como os módulos serão definidos deve ser exaustivamente discutida pelo corpo docente a partir do $\mathrm{NDE}^{14}$ sendo fundamental a participação de egressos do curso e profissionais de saúde que possam contribuir com a proposta. Da mesma forma é importante a inserção de alunos que vivenciam o curso ao longo dos dez períodos, considerando que são os interessados primordiais no desenvolvimento de uma formação sólida e completa.

O desenvolvimento do Módulo integrado deve permitir a utilização de diferentes cenários de aprendizagem ao longo do semestre letivo. Dessa forma, os espaços privilegiados para desenvolvimento dos Módulos integrados configurame pelas salas de aula estruturadas para trabalho tutorial, viabilizando a utilização metodologias ativas de aprendizagem, laboratórios de estudo que permitam etiva articulação da área básica com a área clínica, Unidades Básicas de Saúde do 
SUS, clínica integrada do curso, comunidade, espaços sociais, áreas administrativas do SUS, entre outros.

A construção dos Módulos integrados configura-se como o maior desafio na definição do desenho de currículo ora proposto uma vez que exige dos professores um exercício que o tira da sua zona de conforto, representada pela disciplina que tradicionalmente ministra, estimulando o desenvolvimento docente permanente ${ }^{15}$. Tal perspectiva deve sair de sua formação específica, para discutir com outros professores de diferentes áreas de conhecimento, as estratégias que melhor contribuiriam para a construção de conhecimento pelo aluno, a partir de metodologias ativas de aprendizagem e acompanhadas por um processo permanente de avaliação.

Para viabilizar essa proposta é também fundamental repensar a postura do professor, o qual passa a desenvolver a perspectiva de um tutor, devendo ser responsável por um grupo de até 10 alunos, ao longo de todo o semestre letivo. Isso viabiliza um contato constante do tutor com seu grupo de alunos, fortalecendo o vínculo essencial para as relações de confiança e aprendizado mútuo.

A Extensão curricular (10\% da carga horária total) configura-se por atividades que articulam a inserção da comunidade no aprendizado dos alunos, trazendo benefícios para o entorno do curso, permitindo o reconhecimento de distintas realidades de vida, da ruptura do modelo tradicional de ensino, viabilizando o relacionamento entre aluno, universidade e sociedade ${ }^{16}$. Essas atividades devem ser articuladas a partir da identificação de problemas concretos da comunidade que serão encaradas como desafio para a turma buscar a sua solução até o final do curso. Para essas atividades podem ser articuladas atividades centradas em determinados grupamentos populacionais ou instituições, permitindo tanto o desenvolvimento de atividades de promoção de saúde como de sistência odontológica, permitindo que o aluno desenvolva não somente 
habilidades motoras características da prática odontológica, como possa reconhecer seu papel como agente de transformação da realidade local.

Para a Flexibilização (10\% da carga horária total) estabeleceu-se, como atividades complementares, além das tradicionais (ensino, pesquisa e extensão), a aquisição de novas competências linguísticas, os estágios extracurriculares, projetos multidisciplinares e atividades de cultura e arte. Essas atividades devem ser mediadas por um orientador acadêmico e desenvolvidas desde os semestres iniciais do curso. O orientador acadêmico tem a função primordial de ser um tutor para o aluno colaborando com a coordenação e o NDE na apresentação aos estudantes do projeto pedagógico do curso fazendo seu acompanhamento desde o ingresso ao curso contribuindo no planejamento de seu fluxo curricular, compatível com seus interesses e possibilidades de desempenho acadêmico ${ }^{17}$.

Ainda na perspectiva da Flexibilização, devem ser propostos componentes curriculares optativos ${ }^{18}$, preferencialmente a partir da metade do curso, visando permitir que o aluno efetivamente defina o seu percurso formativo singular, considerando sua formação pregressa. Com essa perspectiva, evita-se a ansiedade do aluno em matricular-se em componentes curriculares apenas como uma obrigação da integralização de seus créditos. Deve-se buscar incluir na condição de componentes optativos atividades que permitam o envolvimento do aluno com outras áreas de conhecimento que viabilizem o trabalho interprofissional, assim como componentes curriculares específicos das ciências odontológicas, inclusive com carga horária de atividades práticas. Esses componentes permitem que o aluno, em articulação com os professores, construam aprendizados que podem ser revisitados futuramente em outros processos de educação permanente. Talvez resida aqui uma das maiores riquezas do currículo integrado, viabilizando o estímulo à autonomia do aluno.

O Trabalho de Conclusão de Curso ${ }^{19}$ (2,5\% da carga horária total) deve ser senvolvido de forma transversal desde o primeiro semestre do curso, podendo 
caracterizar-se por componentes curriculares específicos relacionados ao aprendizado das metodologias da pesquisa científica (com nomenclatura correspondente aos conteúdos a serem aprendidos) ou nos próprios módulos integrados, instrumentalizando o aprendizado baseado em problemas, de forma compatível com o semestre letivo. Os elementos da pesquisa, na perspectiva da graduação, devem ser trabalhados com aumento de complexidade crescente, de forma que nos semestres iniciais o aluno possa se aproximar de atividades menos complexas (levantamento bibliográfico, normas da redação científica, elementos de um projeto de pesquisa), até atingir construções mais complexas (compreensão dos fundamentos filosóficos da ciência, desenhos de estudo, entre outros). Caso as atividades relacionadas à metodologia da pesquisa científica sejam incorporadas nos Módulos integrados, elas contribuirão ainda mais para a integração do currículo, uma vez que seus elementos podem ser aprendidos coerentemente aos problemas desenvolvidos ao longo do semestre.

O Estágio curricular supervisionado deve ser desenvolvido nos $9^{\circ}$ e $10^{\circ}$ períodos, permitindo a efetiva inserção do aluno no Sistema Único de Saúde, desenvolvendo atividades na própria realidade dos serviços, com preceptoria de profissionais da rede ${ }^{20}$, que permita o efetivo aprendizado do aluno a partir da realidade ${ }^{21}$. A formação dos preceptores deve ser preferencialmente intermediada pelo NDE do curso de Odontologia, permitindo coerência com o projeto pedagógico.

O Estágio curricular deve ser considerado momento estratégico tanto para aproximação da IES aos serviços de saúde, como campo privilegiado na educação permanente dos profissionais de saúde ${ }^{22}$. As possíveis limitações dos serviços devem servir muito mais como estímulo para as iniciativas dos cursos em transformar essa realidade do que levar o aluno a desenvolver ações em cenários dversos. Assim, a parceria deve servir tanto para que o aluno aprenda, como para os serviços se aperfeiçoem. É responsabilidade da IES contribuir para o 
fortalecimento das lutas dos profissionais de saúde (muitos egressos delas próprias) por condições dignas de assistência odontológica para a população, compreendendo a perspectiva da saúde como direito de todos e dever do estado.

Fundamental estabelecer ao longo do currículo, "áreas verdes" nas quais os alunos possam dedicar tempo para estudo individual e atividades de sua vida cotidiana (lazer, esporte, cultura) ${ }^{23}$. Essas áreas somente são possíveis quando os horários previstos para o desenvolvimento dessas atividades sejam "permitidos", evitando intervalos muito curtos entre atividades curriculares, o que dificulta o planejamento dessas ações pelos alunos. Para essas áreas também é importante o envolvimento das entidades representativas dos alunos (centros ou diretórios acadêmicos) que busquem o desenvolvimento de atividades de formação política, articulação estudantil, atividades culturais e científicas.

Para viabilizar o desenvolvimento desse currículo, conforme já indicado, é fundamental a utilização de metodologias ativas de aprendizagem em todos os eixos previstos, permitindo que o aluno construa seu aprendizado, com base no princípio de "aprender a aprender" 24.

Da mesma forma, para coerência no desenvolvimento do processo de aprendizado proposto, é fundamental um processo de avaliação ${ }^{25}$, que contribua efetivamente com uma relação entre professor e aluno mediada pela busca de conhecimento, permitindo que ambos sejam sujeitos no processo.

\section{Conclusões}

Deve-se ressaltar que o presente estudo não se propõe a definir uma "receita" para construção curricular, mas deve ser entendido como um exercício que pode contribuir com princípios da formulação de um currículo integrado, o qual, tretanto, deve ser adequado às situações específicas de cada curso de odontologia cada instituição de educação superior, respeitando suas especificidades e rmatizações. 


\section{ciência}

Os principais desafios para alcance da proposta estão relacionados ao processo permanente de desenvolvimento docente, que permita ao professor se apropriar de referenciais pedagógicos contemporâneos na construção de uma nova sala de aula, contemplada pela perspectiva de se pensar a integração como elemento fundamental no processo reflexivo do aluno. Para isso é fundamental, ainda, a formatação de processos avaliativos que não tenham como principal característica a perspectiva somativa e certificadora ainda hoje vivenciada nas Instituições de Educação Superior brasileiras.

A principal limitação do presente estudo configura-se pelo não aprofundamento sobre o percurso a ser trilhado em cada semestre em cada um dos eixos. Entretanto, considerando que o momento fundamental para essa construção coletiva do projeto pedagógico deve ser realizado por gestores, professores, alunos e funcionários, espera-se que o presente estudo possa estimular as reflexões propostas, assim como jogue luzes nessa discussão tão cara aos cursos de Odontologia que se propõem a formar um cirurgião-dentista com responsabilidade social e efetivamente envolvido com os problemas da população brasileira. 


\section{Referências}

1. Costa DAS, Silva RF, Lima VV, Ribeiro ECO. Diretrizes curriculares nacionais das profissões da Saúde 2001-2004: análise à luz das teorias de desenvolvimento curricular. Interface (Botucatu). 2018; 22(67):1183-95.

2. Brasil. Conselho Nacional de Educação Superior, Câmara de Educação Superior. Resolução CNE/CES nº 3, de 19 de fevereiro de 2002. Institui Diretrizes Curriculares Nacionais do Curso de Graduação em Odontologia. 2002.

3. Noro LRA, Roncalli AG, Medeiros MCS, Farias-Santos BCS, Pinheiro IAG. Relação entre conteúdos das disciplinas de curso de odontologia e os ENADE 2004/2010. Avaliação (Campinas). 2017; 22(1):125-39.

4. Moraes BA, Costa NMS. Compreendendo os currículos à luz dos norteadores da formação em saúde no Brasil. Rev. esc. enferm. USP. 2016; 50(spe): 9-16.

5. Noro LRA, Farias-Santos BCS, Sette-de-Souza PH, Pinheiro IAG, Borges REA, Cruz RKS, Diniz RVZ. Basic Cycle in Dentistry Students' Training: The Gap in the Integration of Knowledge and Social Reality. Revista Electrónica de Investigación Educativa. 2018; 20(3):11-8.

6. Franco ECD, Soares NA, Gazzinelli MF. Macro and micropolitics recontextualization of an integrated curriculum: experienced itinerary in nursing undergraduate. Esc. Anna Nery. 2018; 22(4): e20180053.

7. Einzle, MRS, Bagnato MHS. Recontextualização do currículo integrado na formação médica. Pro-Posições. 2015; 26(3):225-38.

8. Souza PA, Zeferino AMB, Da Ros MA. Currículo integrado: entre o discurso a prática. Rev. bras. educ. med. 2018; 35(1):20-5.

Toassi RFC, Stobäus CT, Mosquera JJM, Moysés SJ. Currículo integrado no sino de Odontologia: novos sentidos para a formação na área da saúde. Interface otucatu). 2012; 16(41):529-44. 


\section{ciêncíncia
pural}

10. Brasil. Parecer CNE/CES n ${ }^{o}$ 8/2007, de 31/01/2007. Dispõe sobre carga horária mínima e procedimentos relativos à integralização e duração dos cursos de graduação, bacharelados, na modalidade presencial. Brasília, 2007.

11. Brasil. Lei $\mathrm{n}^{\mathrm{o}}$ 13.005, de 25 de junho de 2014. Aprova o Plano Nacional de Educação - PNE e dá outras providências. Brasília, 2014.

12. Villardi ML, Cyrino EG, Berbel NAN. A metodologia da problematização no ensino em saúde: suas etapas e possibilidades. In: A problematização em educação em saúde: percepções dos professores tutores e alunos. São Paulo: Editora UNESP; São Paulo: Cultura Acadêmica, 2015, pp. 45-52.

13. Lazzarin HC, Nakama L, Cordoni Junior, L. Percepção de professores de odontologia no processo de ensino-aprendizagem. Ciênc. saúde coletiva. 2010; 15(supl.1):1801-10.

14. Brasil. Comissão Nacional de Avaliação da Educação Superior. Resolução n ${ }^{\circ}$ 01, de 17 de junho de 2010. Normatiza o Núcleo Docente Estruturante e dá outras providências. Brasília, 2010. Acessado em 25 de outubro de 2018. Disponível em http://www.ceuma.br/cpa/downloads/Resolucao_1_2010.pdf.

15. Lage RH, Almeida SKTT, Vasconcelos GAN, Assaf AV, Robles FRP. Ensino e Aprendizagem em Odontologia: Análise de Sujeitos e Práticas. Rev. bras. educ. med. 2017; 41(1):22-9.

16. Fadel CB, Bordin D, Kuhn E, Martins LD. O impacto da extensão universitária sobre a formação acadêmica em Odontologia. Interface (Botucatu). 2013; 17(47):937-46.

17. Universidade Federal do Rio Grande do Norte. Resolução n ${ }^{\circ}$ 171/2013CONSEPE, de 5 de novembro de 2013. Aprova o Regulamento dos Cursos Regulares de Graduação da Universidade Federal do Rio Grande do Norte. Natal, 13. 


\section{ciênncia
piural}

18. Medeiros CCBM, Alves MCSF, Medeiros Júnior A, Reis MKS, Silva LJA, Tertulino MD. Educational opportunities for reflective practice. RGO, Rev. Gaúch. Odontol. 2015; 63(4):418-25.

19. Silva Júnior MF, Assis RIF, Sousa HA, Miclos PV, Gomes MJ. Iniciação científica: percepção do interesse de acadêmicos de odontologia de uma universidade brasileira. Saude soc. 2014; 23(1):325-35.

20. Lima PAB, Rozendo CA. Desafios e possibilidades no exercício da preceptoria do Pró-PET-Saúde. Interface (Botucatu). 2015; 19(supl. 1):779-91.

21. Leme PAT, Pereira AC, Mehghim MC, Mialhe FL. Perspectivas de graduandos em odontologia acerca das experiências na atenção básica para sua formação em saúde. Ciênc. saúde coletiva. 2018; 20(4):1255-65.

22. Werneck MAF, et al. Nem tudo é estágio: contribuições para o debate. Ciênc. saúde coletiva. 2010; 15(1):221-31.

23. Basso C, Graf LP, Lima FC, Schmidt B, Bardagi MP. Organização de tempo e métodos de estudo: Oficinas com estudantes universitários. Rev. bras. orientac. prof. $2013 ; 14(2): 277-88$.

24. Garcia O, Néstor S. El pensamiento complejo y la transdisciplinariedad: fenómenos emergentes de una nueva racionalidad. Rev.fac.cienc.econ. 2012; 20(1):269-91.

25. Gonçalves MR, Ney MG. Contribuições da avaliação mediadora para a melhoria da qualidade da Educação. Agenda Social. 2010; 4(2):96-8.

abmetido: 10/01/2019

provado: 30/04/2019 